\title{
Comparing Electronic and Traditional Lab Notebooks in the Advanced Lab
}

\author{
Melissa Eblen-Zayas* \\ *Department of Physics and Astronomy, Carleton College, 1North College St., Northfield, MN 55057
}

\begin{abstract}
Two years ago, the advanced lab course at Carleton College switched to using electronic lab notebooks (ELNs) instead of traditional paper lab notebooks. In the wake of this change, a survey of students in the class shows that over $80 \%$ of the students would recommend other science classes use ELNs. The survey also highlights what students perceive as strengths and weaknesses of each notebook format. This paper discusses the student impressions of the relative merits and drawbacks of ELNs as well as providing an overview of the reasons for switching to ELNs and the considerations in selecting ELN software.
\end{abstract}

Keywords: electronic lab notebook, advanced physics laboratory instruction, instructional technology for labs.

PACS: 01.40.-d; 01.50.Lc

\section{INTRODUCTION}

Keeping a record of laboratory work is important in professional lab settings. To encourage student development of good record-keeping habits, curricular labs often require that students maintain a lab notebook. In the AAPT Recommendations for the Undergraduate Physics Laboratory Curriculum, ${ }^{1}$ two of the six main learning outcome focus areas (Developing Technical \& Practical Laboratory Skills and Communicating Physics) include specific goals related to lab notebooks.

The use of electronic lab notebooks (ELNs) is expanding in a variety of professional settings.,3 ELNs allow for easier management and organization of data in electronic form as well as facilitating collaboration among groups of researchers; however, portability and ease of entering sketches continue to be advantages of paper lab notebooks. For the past two years, students in the advanced lab course at Carleton College have used ELNs. This paper discusses the reasons for switching to ELNs, the criteria considered when selecting ELN software, the way in which ELNs were introduced and used in the course, and student reactions to using electronic lab notebooks.

\section{COURSE DESCRIPTION}

Phys 342 Contemporary Experimental Physics is a ten-week advanced laboratory class, typically taken by physics majors in the junior year. Prior to taking this course, students have taken at least two intermediatelevel lab classes, including one in which keeping a traditional lab notebook is a significant portion of the lab grade. The advanced lab course includes class meetings three times per week and a weekly four-hour lab. Students are expected to work on their experimental projects outside of the designated lab time as well. The laboratory component of the course consists of three two-week labs that are designed by the instructor, followed by a four-week, studentdesigned project. All experimental work in the lab is carried out in groups of two or three.

\section{ELN SELECTION}

Perceived benefits for both students and instructors led to the adoption of ELNs in the advanced lab course. For students, collaboration is easier with ELNs; students in a lab group can share their lab notebooks with each other and comment on each other's notebook entries. For the instructor, ELNs make it easier to keep track of what students are doing. Particularly in the advanced lab where projects can extend over many weeks and much of the work takes place outside of designated lab hours, it is inconvenient to collect individual lab notebooks to track project progress. With ELNs, the instructor can follow project developments in a less intrusive manner. Also, because the software time stamps every entry, the instructor can tell if the lab notebook is a genuine record of the work as it is being done or if the lab notebook was filled out after the fact. Finally, as more work is being done on computers, it makes sense to keep the records electronically, rather than having students print an electronic file to paste in a paper notebook.

Many options exist for implementing electronic notebooks, ranging from Google docs to Evernote to commercial ELNs, and the landscape of available software is changing rapidly. When selecting an ELN, we considered six features. First and foremost,

2015 BFY Proceedings, edited by Eblen-Zayas, Behringer, and Kozminski; Peer-reviewed, doi:10.1119/bfy.2015.pr.007

Published by the American Association of Physics Teachers under a Creative Commons Attribution 3.0 license.

Further distribution must maintain attribution to the article's authors, title, proceedings citation, and DOI. 
students had to be able to easily, and selectively, share portions of their notebooks with others in the class. In addition, the notebooks needed to allow the instructor or peers to comment on entries in a student's notebook. Second, since students might use a variety of devices and operating systems to access the ELNs, the software had to work well across different platforms. Third, the software needed to provide an opportunity for the instructor to distribute some background materials about the labs at the beginning of the term and to push additional materials to the student lab notebooks during the course, without overwriting student entries in the lab notebooks. Fourth, because students often used their phones to capture photos of experimental set-ups or sketches on a whiteboard, software with a phone app allowing photos to be easily imported to the notebook was desirable. Fifth, the software should allow quick and easy typesetting of equations. Finally, we did not want students to pay much more for the ELN than they would have for a traditional paper lab notebook. After comparing features among three ELNs (Microsoft OneNote, LabArchives, and PerkinElmer Elements) and talking with other ELN users, LabArchives was chosen as the ELN for the course. ${ }^{4}$

\section{ELN IMPLEMENTATION}

An in-class activity on the first day of the course, based on activities developed by others, ${ }^{5}$ provided an introduction to lab notebooks and the possible benefits and drawbacks of ELNs. Students, working in groups of two or three, were given several pages from the lab notebooks of famous scientists. After examining the lab notebook pages, they were asked to identify what types of things were recorded, the style and tone of the notes, and similarities and differences between the various notebook pages. Through the discussion, students developed an understanding that lab notebooks serve two different audiences - the individual investigator and collaborators or external observers who want to understand or reproduce the work.

The class discussion then moved to the limitations of paper lab notebooks and the potential trade-offs involved in using an electronic lab notebook versus a paper lab notebook. Students immediately identified the potential for easy sharing of electronic lab notebooks with remote collaborators as a major benefit. In addition, having just struggled to decode the poor handwriting of several famous scientists, students also identified legibility of the record as a benefit of an ELN. At the same time, students recognized the limitations of technology. In particular, they raised questions about the permanence of an electronic lab record and concerns about the ease of the user interface. Upon wrapping up the discussion, the key elements of LabArchives were demonstrated in class to make students aware of the features and functions of the ELN software.

Students were expected to keep a complete record of all their lab work in LabArchives. When the students first logged in, they found a class notebook that already had some structure to it. Each of the instructor-designed experiments had its own folder within the notebook and there was a final project folder. The folders for the instructor-designed labs were populated with four sub-folders: one subfolder with background materials (introduction to the labs, descriptions of the experimental set-up/equipment, relevant papers from the literature) and three additional subfolders -- one for student notes on equipment/procedures, one for data files and associated notes, and one for notes on analysis/conclusions. Many students added additional subfolders to the provided folders, while a few students chose to create their own file organization system.

During the first half of the course when working on the instructor-designed labs, students received written feedback on their lab notebook after each of the twoweek experiments (three times total). In addition, the instructor monitored the lab notebooks for early identification of any students who were not providing an adequate record of their work. During the openended projects in the second half of the course, the lab notebooks provided the instructor a window into project progress that was accessible at any time. Student notebooks were again evaluated at the end of the final project. Evaluation of the lab notebook contributed to $10 \%$ of the overall course grade. Having the lab notebook carry weight in the overall course grade encourages students to consider the importance of good record-keeping habits and reinforces that the process of organizing one's thoughts and data is an important part of lab work. At the end of the course, students could export their LabArchives notebook as a PDF, but we did not track how many students did this.

\section{STUDENT RESPONSE}

In the last week of the course, students were given an in-class survey asking about LabArchives and ELNs. The survey consisted of six rating scale questions about using the LabArchives ELN, three free response questions about LabArchives, and three general questions about past, present, and future use of ELNs. None of the 22 students in the course had used an ELN prior to this course, although all students had taken a course that required keeping a traditional paper lab notebook. Over half the students reported that they kept a better record of their lab work with an ELN than with a traditional paper notebook, and few students felt their record keeping was significantly worse with the 
ELN (Fig.1). Additionally, most students judged that it took less time to enter information into an ELN as compared to a paper lab notebook (Fig. 2). Student approaches to recording work in the lab notebook varied by group. Some groups would have as few as four or five lab notebook entries for a day of work, while other groups would have more than 30 entries. To some extent this reflects a difference in opting for fewer, longer entries versus many shorter entries, but it also reflects the variation in how engaged the students were as record keepers. Additional analysis of the numbers and types of lab notebook entries and how that relates to student learning is an area for future research.

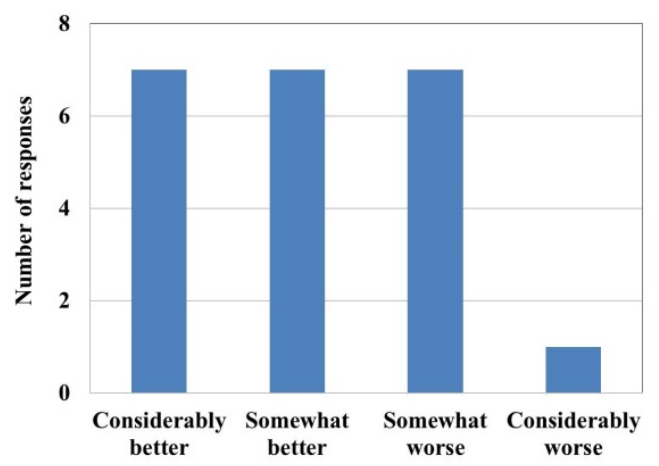

FIGURE 1. Student responses to the question, "Do you keep a better or worse record of your laboratory experiment with LabArchives as compared to a traditional lab notebook?”

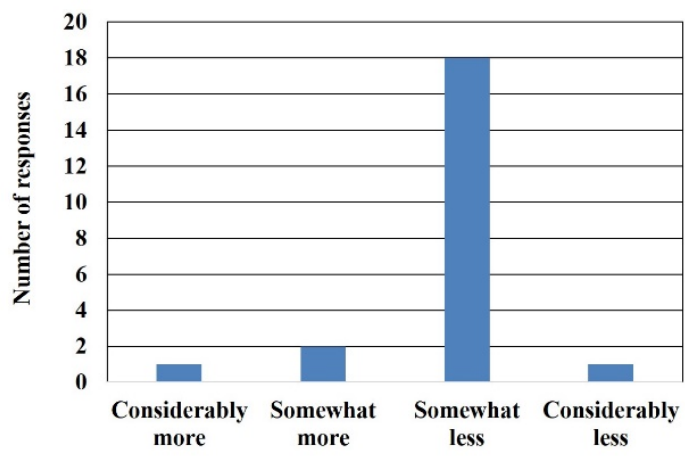

FIGURE 2. Student responses to the question, "Does it take you more or less time entering information into LabArchives as compared to a traditional lab notebook?”

When asked to identify what aspects of record keeping were easier with an ELN, the two most common responses were easier collaboration (8 students) and easier integration of digital graphics and photos (11 students). The list of ELN benefits mentioned by at least two or more students included:

- Easier collaboration with group members;
- Easier integration of digital graphics and photos;

- Storing many different file formats easily;

- Easier organization of files, and the ability to reorganize later;

- Accessibility from any device, anywhere;

- Ability to link to external on-line resources;

- Easier to read notes of those with illegible handwriting.

One of the unexpected benefits of ELNs was that over $85 \%$ of students felt less effort was required to organize information in an ELN than in a paper notebook (Fig. 3). Students frequently mentioned that they appreciated the ease with which the information and files could be rearranged as needed. As one student noted, "You can rearrange files to organize information after putting it in the notebook." Another student wrote, "I particularly enjoyed that I could divide my work into sections. This helped me to organize my thoughts better." However, not all students saw the benefits of the flexible organizational structure of the ELN. One student wrote, "It's not clear how to organize the notebook because 1) we can add lots of entries to a page or 2) we can add lots of pages to a folder.”

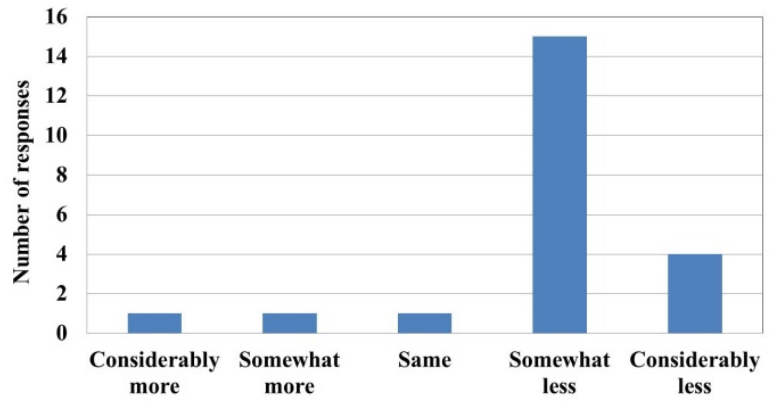

FIGURE 3. Student responses to the statement, "The effort it required to organize the information in LabArchives compared to a traditional notebook was..."

When asked to identify what aspects of the record keeping were more difficult with an ELN as compared to traditional lab notebooks, the most common response was the hassle of including equations or quick sketches (6 students). The list of ELN drawbacks mentioned by at least two or more students included:

- The difficulty of including equations or sketches;

- Technical difficulties;

- Taking more effort to record simple things;

- Having to write notes by hand and transfer them later, if a computer is not present;

- Absence of a hard copy to show someone else or to reference later. 
LabArchives did have some technical issues, particularly with regards to the embedded spreadsheet application, and that was a source of student frustration. Some students avoided the problem by using other spreadsheet software and then uploading files to LabArchives, but nine students (out of 22) somewhat or strongly agreed that technical issues were an impediment to getting work done in the ELN.

An additional factor that may impact student satisfaction with using an ELN is the type of device used to access the notebook. For this course, students could use desktop computers in the labs or personal laptops, tablets, or phones to access the software. This year, none of the students used tablets. Students did use a variety of other device combinations to log on to LabArchives. The survey asked students to estimate the percentage of time during the course that they used a particular device to access LabArchives. The estimates varied widely, with some students indicating they used the desktop computers in the labs for $80 \%$ of their LabArchives access. Other students used personal laptops as much as $90 \%$ of the time. One student used a smart phone for $50 \%$ of his or her LabArchives access. Exploring patterns of device usage and how that impacts student record keeping and the experience with the ELN is another area for future research.

\section{INSTRUCTOR RESPONSE}

From the instructor perspective, student record keeping with LabArchives was of equal or better quality than student record keeping with traditional paper notebooks. The ELN made the aggregation of electronic files related to data collection and analysis straightforward. When using LabArchives, students were more likely to take a photo of an experimental set-up or what they saw on an instrument screen instead of drawing a sketch, as they would in a traditional lab notebook. Photographic records proved helpful when the instructor wanted to revisit aspects of the experiment with a student because the photos often contained key details that were not included in handdrawn sketches.

For the instructor, one of the biggest advantages of using ELNs in the advanced lab was the ability to review student notebooks at any time. Students did not need to turn in their notebooks for the instructor to be able to track student progress. Easy access to the notebooks allowed the instructor to provide better assistance when students encountered difficulties while working in the lab after hours because the instructor could view the notebooks remotely to better understand what type of help students might need.

\section{SUMMARY}

Student response to electronic lab notebooks generally, and to LabArchives in particular, was overwhelmingly positive. $82 \%$ of the students would recommend the use of LabArchives in another science course. When asked if they were given a choice of how to keep records in a research or curricular lab, 14 out of 22 students said they would choose to use an ELN, and 7 additional students said that their choice would depend on the situation. Only one student indicated a preference for traditional lab notebooks in all situations. Of those students who said that their choice would depend on the situation, the biggest factor impacting their decision would be the nature of the collaboration that was required; nearly every student indicated that ELNs were better for collaborative work.

Course instructors also realized significant benefits from using ELNs. In particular, the ability to view any student lab notebook at any time provided a better understanding of how the experimental projects were unfolding, as well as allowing the faculty member to help troubleshoot a project by examining student notes at any time.

Further examination of the nature of the lab notebook entries, the notebook organizational structure, the types of devices used to access the ELN, and how those factors impact student performance in the course might yield interesting clues about the pedagogical value of ELNs, as well as identifying the challenges that this instructional technology poses for students.

\section{ACKNOWLEDGMENTS}

I would like to thank to Janet Russell and Carly Born of Carleton Information Technology Services who helped to design and administer the student survey. I would also like to thank Eric Hazlett who cotaught the advanced lab course this year.

\section{REFERENCES}

1. J. Kozminski, et al. AAPT Recommendations for the Undergraduate Laboratory Curriculum. http://www.aapt.org/Resources/upload/LabGuidlinesDoc ument_EBendorsed_nov10.pdf (November 2014).

2. J. Giles, Nature 481, 430-431 (2012).

3. H. K. Machina and D. J. Wild, Journal of Laboratory Automation 18, 264-268 (2013).

4. http://www.labarchives.com

5. L. J. Atkins and I. Y. Salter, Physics Education Research Conference (PERC) AIP Conference Proceedings 1513, 50-53 (2012). 